\title{
How Dentistry Coped with COVID -19
}

Dr. Komal Waghmare ${ }^{1 *}$, Dr. Gona Shruti ${ }^{2}$, Dr. Supriya ${ }^{3}$, Dr. Babu Waghmare ${ }^{4}$

${ }^{1}$ MDS, Oral \& Maxillofacial Surgery, Mumbai, India

${ }^{2}$ MDS, Oral \& Maxillofacial Surgery, Hyderabad, India

${ }^{3}$ MDS, Prosthodontics \& Implantology, Mumbai, India

${ }^{4} \mathrm{MDS}$, Orthodontics and Dentofacial Orthopedics, Mumbai, India

DOI: $10.36347 /$ sjds.2020.v07i11.002

| Received: 16.10.2020 | Accepted: 03.11.2020 | Published: 16.11.2020

*Corresponding author: Komal Waghmare

Abstract

Review Article

After the detection of $1^{\text {st }}$ few cases of SARS COV-2 in Wuhan city in China in late 2019 little did we know that it will have such a gripping effect all over the globe. Health care workers were challenged in giving optimum services to the patients infected with Covid-19 and at the same time prevent cross infections. Since the spread has been rampant through nasopharyngeal and salivary secretions providing dental care to on going patients or emergency cases, had to meet with changes in routine standard operating procedures. How dentistry tried to combat the new changes by modifying regular practice will be discussed in this article.

Keywords: SARS COV-2, COVID-19 Virus Pandemic, Emergency Dental Practice, PPE, Infection Control, Dental Practice Management.

Copyright $\odot 2020$ The Author(s): This is an open-access article distributed under the terms of the Creative Commons Attribution 4.0 International License (CC BY-NC 4.0) which permits unrestricted use, distribution, and reproduction in any medium for non-commercial use provided the original author and source are credited.

\section{INTRODUCTION}

Pandemics inflict devastating consequences on communities and cause long term rippling effects in the economy and health care system. Before the AIDS pandemic in 1981 dentists did not commonly wear masks or eye protection. In the late 1980s and early 1990s, in an attempt to protect health care workers, the Occupational safety, health Administration (OSHA) and the $\mathrm{CDC}$ proposed guidelines to reduce exposure to blood borne pathogens such as HIV and Hepatitis B. By its nature, dentistry is an intimate occupation. The dentists works within an inch of a patient's body [1]. At the end of 2019, the first cases of a pulmonary disease of unknown aetiology were detected in Wuhan City, China. In the following months, this new pathogen spread throughout Europe and then worldwide; in March 2020, the World Health Organization (WHO) officially declared a pandemic alert [2].

During this challenging time we are in a position of balancing interests of the patients, office staff members and entire community. To promote the greatest good for greatest number, providing limited services to only those with urgent needs is supported by the principle of Beneficence [3].
Pathophysiology and spread of the COVID-19 virus

This new virus, highly infective especially through airborne transmission, is responsible for an acute respiratory syndrome, distinguished by an often asymptomatic, but potentially lethal, interstitial bilateral pneumonia [4].

1. The most common receptor involved in the viruscell interaction is angiotensin- converting enzyme 2 (ACE- 2), which is present at high concentrations in lungs, myocardial cells and kidney, as well as on oral mucosa (especially of the salivary glands and tongue)[ 5,6] The incubation period of Sars- CoV- 2 varies between 3 and 14 days; however, a 24- day incubation period has also been reported dyspnea [7].

2. In most instances, the infection brought on by this new coronavirus is asymptomatic or causes few symptoms [8].

Infected patients mainly exhibit night fever, dry cough, sore throat and asthenia; patients with more severe disease can exhibit dyspnea. US Centers for Disease Control and Prevention (CDC) recently included 'sudden loss of taste (dysgeusia/ageusia) and smell (anosmia/hyposmia)' as symptoms of COVID-19. 


\section{Management of patients in the initial phases of the} pandemic

Based on the experience gained from the previous outbreak of SARS-CoV and the data available on SARS-CoV-2 and its associated disease (COVID19), certain specific measures were discussed for dental patient management in this pandemic period of COVID-19 (summarized in Fig-1) [9].

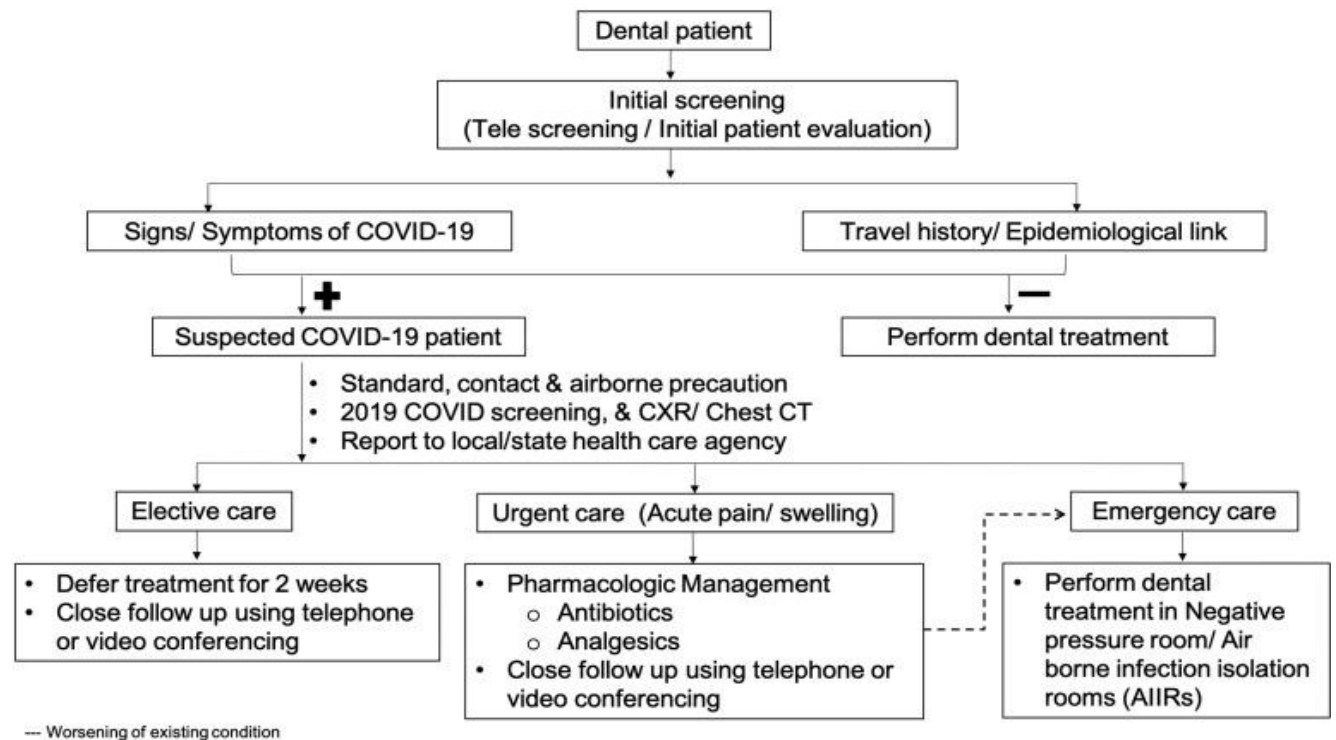

Fig-1

Patients coming to dental clinics during the pandemic were considered as either asymptomatic carriers or were either considered as asymptomatic patients, carriers or patients in their recovering phase and thus practioners had to adhere to following protective measures.

\section{Introduction of personal protective kit in dentistry}

The high virulent nature of SARS COV-2 made the Personal protective kit mandatory for routine procedures during the pandemic. This was to provide highest level of protection both for the patient and the dentist. The components of the PPE included Gowns or Jump- Suits so as to provide protection from head to toe, Respirators, Goggles for eye protection, Faceshields and gloves.

\section{Gowns/Jumpsuits}

According to CDC these had to be nonabsorbent so that the aerosols or any secretions do not directly touch the skin of the operator and covered the operator almost from head to toe. Donning and doffing areas of these were supposed to be separate from the operating areas. A vigorous hand washing regime were to be followed before and after the procedure. The gowns/ jumpsuits had to be changed after every patient to avoid cross- contamination.

\section{Respirators}

1. Suddenly the surgical masks were rendered useless. Since the air droplet COVID- 19 particles are estimated to be $0.06-0.14 \mu \mathrm{m}$ in diameter, the most efficient masks are presumed to be FFP2/N95, FFP3/N99 and N100. Surgical masks, however, remain valid devices for all procedures that do not create an aerosol [10].

2. FFP3/N99 and N100 facepieces without valves seem to be the devices primarily indicated to guarantee the highest level of protection for both operator and patient, but it is quite challenging to achieve normal air exhalation when these facepieces are used for an extended period of time [11].

In dental procedures, the mask should be considered as disposable and the mean surgical period does not exceed 2 hours; therefore, it is suggested to use a mask with the highest filtration efficacy without a valve, or a valved mask covered by a surgical mask [2].

\section{Eye Protection}

In the dental field, eye protection has been consistently indicated to minimise contact of the eyes with mechanical (e.g., slivers and foreign bodies), chemical (e.g., acids and disinfectants) and biological (e.g., saliva, blood, oral fluids) agents [12]. The ocular pathway is known to be one of the most frequent routes of infection with Sars- CoV- 2 [13].

Eyewear with enveloping frames had to be used and having wide lenses to cover the face as much as possible. Alternatively, plastic shields are also preferred to glasses because of their greater capacity to protect the face from aerosol droplets. These shields could be worn directly on the forehead or could be included in the surgical mask [14]. 


\section{Dental Emergency Cases}

Dental emergencies, according to the ADA, "are potentially life threatening and require immediate treatment to stop on going tissue bleeding or to alleviate severe pain or infection." These conditions include uncontrolled bleeding; cellulitis or a diffuse soft tissue bacterial infection with intraoral or extraoral swelling that potentially compromises the patient's airway; or trauma involving facial bones that potentially compromises the patient's airway. Dentists should use their professional judgment in determining a patient's need for urgent or emergency care [15]." As part of the emergency guidance, the Association added urgent dental care which "focuses on the management of conditions that require immediate attention to relieve severe pain and/or risk of infection and to alleviate the burden on hospital emergency departments."

Examples of urgent dental care treatments, which should be treated as minimally invasively as possible, include:

- Severe dental pain from pulpal inflammation.

- Pericoronitis or third-molar pain.

- Surgical postoperative osteitis or dry socket dressing changes.

- Abscess or localized bacterial infection resulting in localized pain and swelling.

- Tooth fracture resulting in pain or causing soft tissue trauma.

- Dental trauma with avulsion/luxation.

- Dental treatment cementation if the temporary restoration is lost, broken or causing gingival irritation

Other emergency dental care includes extensive caries or defective restorations causing pain; suture removal; denture adjustments on radiation/oncology patients; denture adjustments or repairs when function impeded; replacing temporary filling on endo access openings in patients experiencing pain; and snipping or adjustments of an orthodontic wire or appliances piercing or ulcerating the oral mucosa [15].

Dr. Donald F. Cohen et al., recommended to carry out treatments for on record patients suffering from dental emergencies rather than sending them to emergency department, public health clinic or dental school which were already overburdened during this pandemic. They proposed to use personal protective equipment for patients with urgent needs only, during the pandemic not only to conserve a limited supply of PPE but as also to reduce the community exposure and exposure of health care workers. Although shutting down the clinics or limiting the practice was causing a financial constraint on private health care workers as well worsening dental problems due to delay in treatment the greater good had to be taken into consideration [3].

\section{Telecommunication/teledentistry/telescreening}

A new method had to be adopoted to help out to patients who were suspected covid-19 patients or in areas where clinics were shut down completely i.e Teledentistry. It is "a combination of telecommunications and dentistry, involving the exchange of clinical information and images over remote distances for dental consultation and treatment planning" [16].

\section{Telescreening}

The questionnaire to screen the patients would include [9].

- Exposure to a known covid-19 positive patient

- Any recent travel history to an area with high incidence of covid-19

- Presence of any symptoms such as fever or cough

A positive response to any of these questions meant delaying of the elective dental procedure for atleast 2 weeks. These patients were to be encouraged to engage in self quarantine and contact their primary health care physician by telephone or email.

Nonemergency dental procedures had to be then carried out after telescreening and telecommunication. According to the ADA Association, these procedures include but are not limited to:

- Initial or periodic oral examinations and recall visits, including routine radiographs.

- Routine dental cleaning and other preventive therapies.

- Orthodontic procedures other than those to address acute issues (e.g., pain, infection, trauma).

- Extraction of asymptomatic teeth.

- Restorative dentistry including treatment of asymptomatic carious lesions.

- Aesthetic dental procedures.

Guidelines (as of May, 29, 2020) recommend that elective care for dental patients with confirmed or suspected COVID-19 be deferred until the patient meets criteria for discontinuation of home isolation [17]. The American Dental Association (ADA), (American Dental Association ADA Interim Guidance for Management of Emergency and Urgent Dental Care. Chicago, Ill: 2020 [18]. The Occupational Safety and Health Administration (OSHA), (Occupational Safety and Health Administration Dentistry Workers and Employers. Washingto, DC: United States Department of Labor 2020 [19] and others to prevent spread of infection. Dental care providers were advised to:

1. If possible, perform procedures in a negative pressure room, (airborne infection isolation room or AIIR) for aerosol-generating dental procedures.

2. Follow standard, contact, and airborne precautions including hand hygiene practice. 
3. Consider taking extraoral radiographs instead of intraoral (e.g. panoramic radiography) to avoid gag reflex.

4. Limit the number of dental healthcare providers (DHCP) present during the procedure to only those essential for patient care and procedure support. Visitors should be limited to those who are necessary.

5. Use a dental hand-piece with anti-retraction function, four-handed dentistry, high evacuation suction and rubber dams to minimize droplet splatter and aerosol generation.

6. Minimize the use of ultrasonic instruments, highspeed handpieces, and 3-way syringes.

7. Perform endodontic procedures with dilute (1\%) solutions of sodium hypochlorite to extend supplies without adverse effects on outcomes.

8. Use resorbable sutures (i.e. sutures that last 3 to 5 days in the oral cavity) to eliminate the need for a follow up appointment.

9. Disinfect surfaces with EPA-approved chemicals and maintain a dry environment.

10. Wear an N95 or equivalent or higher-level respirator such as a disposable filtering facepiece respirator, a powered air-purifying respirator, or an elastomeric respirator; eye protection (e.g. goggles, reusable face shields); gloves; and a gown.

Using a negative pressure room may create challenges as most of the private dental offices lack AIIRs and larger dental organizations may have dental operatories that are clustered in open spaces without physical barriers. As an alternative, CDC is currently recommending placing a portable HEPA air filtration unit within the vicinity of the patient's chair (but not behind the DHCP) while the patient is actively undergoing, and immediately following, a potentially aerosol-generating procedure [20].

\section{Adaptations in dental setups}

Dental setups invariably carry the risk of COVID-19 infection due to specificity of its procedure (aerosol production), proximity to the oropharyngeal region and frequent exposure to the saliva. Moreover if adequate precautions is not taken, the dental office can potentially expose patients to cross contamination. Thus the following protocols were proposed [21].

\section{Waiting Area}

a) Display of visual alerts at the entrance of the clinic and reception area about respiratory hygiene, cough etiquette, social distancing, and disposal of contaminated items in trash cans [22].

b) As soon as the patient enters the reception area, ask them to wash their hands using hand wash or alcohol- based hand rub. Use tissue paper or hand dryer to dry the hands instead of towels. Tissue paper dispenser and foot- operated waste bin are mandatory [22]. c) Include temperature recordings as part of your routine patient assessment before performing any dental procedure. A non-contact forehead thermometer can be used to measure the patient's body temperature [13]. Patients presenting with fever or respiratory disease/ symptoms were to be registered and referred to designated hospitals.

L. Ondine, $\mathrm{T}$ Dorottya et al., in their prespective article, Oral health care during the COVID19 pandemic had laid down these following guidelines for effective control of infection in dental office [23].

- Appointments to be scheduled apart to minimize the contact between the patients. Patients can wait in their personal vehicle, until its their turn.

- All reading materials and magazines to be removed from the dental office.

- Aerosol generating procedures should be scheduled at the end of the program.

- Handpiece must be sterilized after each patient

- After providing dental care, facial protective equipment should be cleaned and disinfected.

- The x-ray equipment, the light and dental chair to be disinfected according to the instructions of manufacturer.

- As intraoral x-ray can induce severe salivary secretion and coughing extraoral radiographies are alternatives

- Frequently used surfaces such as doors, handles, desks, bathrooms must be disinfected often.

- Studies have shown coronavirus could be effectively eliminated in 1 minute when the surfaces were disinfected with $62 \%-71 \%$ ethanol, $0.5 \%$ hydrogen peroxide or $0.1 \%$ sodium hypochlorite [24].

\section{Preprocedural Modifications}

a) Drape the patient preferably with single-use, disposable plastic apron [22].

b) Ask the patient to remove the mask [22].

c) Preprocedural mouth rinse: Effective reduction in salivary microbial load can be achieved by rinsing with $0.2 \%$ povidone-iodine or $1 \%$ hydrogen peroxide before the procedure [25] Studies conclude that chlorhexidine is ineffective against COVID- 19 [13].

\section{Procedural Modifications}

- When handpieces or ultrasonic devices must be used, the use of a rubber dam is indicated as this significantly reduces the amount of aerosol containing saliva and/or blood, providing a $70 \%$ reduction of droplets around the surgical field [26].

- When isolation using a rubber dam is not possible, manual instrumentation is preferred over high- speed handpieces [13]. 
a) Installation of high vacuum extra oral suction devices recommended [21].

b) Maintain natural air circulation within the operatory, through frequent opening of windows and by using an exhaust blower to extract the room air into the atmosphere [21].

c) The window air condition system/split AC should be frequently serviced, and filters cleaned [21].

d) Commercially available electrostatic air conditioner filters can be used. Use of indoor portable air cleaning system equipped with HEPA filter and UV light may be used [21]. Currently, there is no evidence regarding sanitation devices that are specifically effective against Sars- CoV- 2 [2].

e) Use resorbable sutures to eliminate the need of follow-up appointment [27].

f) Extending time between patients, as necessary, to allow for proper cleaning and decontamination of examination rooms [28];

g) Extensive preparation strategies for the physical office and staff to prepare the environment and familiarize the staff with new policies and procedures to reduce the risk of transmission of SARS-CoV-2 [28];

h) Implementing a chairside checklist to prepare operatories [28].

i) Although there is a lack of information concerning environmental sanitation related to coronaviruses, some options are always useful for reducing bacterial and viral loads in dental clinics. Common sense- based guidelines suggest an adequate air change after each dental procedure by opening the windows in surgical rooms and in the waiting room [2].

\section{Waste Management}

The infectious medical and domestic waste of suspected or confirmed COVID- 19 patients should be disposed off in double- layered yellow color bags with gooseneck ligation [13].

The bags should be marked and disposed off in accordance with the Biomedical Waste Management and Handling Rules, 2018 [29].

\section{CONCLUSION}

The COVID-19 situation lead to sudden changes in dentistry both in its practice module and costs of the treatments. The introduction of PPE kits became mandatory for aerosols producing procedures. Many clinics were not equipped with air purification systems like HEPA filters or UV lights, enough space for donning and doffing of PPE kits and sitting arrangements for patients in waiting area. Also not much research data was available regarding the course of the disease and its prognosis in the initial phase of the pandemic. Inspite of which dental professionals had render services atleast the emergency ones making them quite vulnerable to get infected by the virus. Another challenging aspect was to prevent cross-contamination. Teledentistry and triaging, reducing the procedure time and implementation of new sanitization techniques might be few of the probable reasons how we could have combated the situation. Further studies will be required to prove the same. Also will dentistry permanently imbibe these new methods only time can tell.

\section{REFRENCES}

1. Steven H, Ferneini EM. A Pinch of Prevention is worth a Pound of cure: Proactive dentistry in the wake of Covid-19 J. Oral Maxillofacial Surg. 2020 Jun; 78(6):860-861.

2. Checchi V, Bellini P, Bencivenni D, Consolo U. COVID- 19 Dentistry- Related Aspects: A Literature Overview. Int Dental J. 05 July 2020, First published: https://doi.org/10. In1111/idj.12601Citations:

3. Cohen DF, Kurkowski MA, Wilson RJ Jr, Jonke GJ, Dr, Patel OR, Pappas RP. Ethical Practice During The Covid-19)- Am Dent Assoc. 2020 May; 151(5):377-378

4. Meng L, Hua F, Bian Z. Coronavirus Disease 2019 (COVID-19): Emerging and Future Challenges For Dental And Oral Medicine. J Dent Res. 2020: 99(5) 481-487.

5. $\mathrm{Xu} \mathrm{H}$, Zhong L, Deng J. High Expression of ACE2 Receptor of 2019- Ncov on the Epithelial Cells of Oral Mucosa. Int J Oral Sci. 2020; 12:8.

6. Yan R, Zhang Y, Li Y. Structural basis for the recognition of SARS- CoV- 2 by full- length human ACE2. Science. 2020; 367:1444-1448.

7. Balla M, Merugu GP, Patel M. COVID- 19, Modern Pandemic: A Systematic Review from Front- Line Health Care Providers' Perspective. J Clin Med Res. 2020; 12: 215- 229.

8. Chen N, Zhou M, Dong X. Epidemiological and Clinical Characteristics of 99 Cases of 2019 Novel Coronavirus Pneumonia in Wuhan, China: A Descriptive Study. Lancet. 2020 395:507-513.

9. Ather A, Patel B, Ruparel NB, Diogenes A, Hargreaves KM. Coronavirus Disease 19 (COVID-19): Implications for Clinical Dental Care. JOE. 2020; 46(5):584-595.

10. Zhu N, Zhang D, Wang W. A Novel Coronavirus from Patients with Pneumonia in China, 2019. N Engl J Med. 2020; 382:727-733.

11. Verbeek JH, Rajamaki B, Ijaz S. Personal Protective Equipment For Preventing Highly Infectious Diseases Due To Exposure To Contaminated Body Fluids In Healthcare Staff. Cochrane Database Syst Rev. 2020; 4: CD011621.

12. Checchi L, Montevecchi M, Violante F. Management rules for a dental practice: biological risk and safety at work. Dental Cadmos. 2012; 3:140-156.

13. Peng $\mathrm{X}, \mathrm{Xu} \mathrm{X}, \mathrm{Li} \mathrm{Y}$. Transmission routes of 2019- $\mathrm{nCoV}$ and controls in dental practice. Int $\mathrm{J}$ Oral Sci. 2020; 12:9. 
14. Montevecchi M, Checchi V, Felice P. Management rules of the dental practice: individual protection devices. Dental Cadmos. 2012; 80: 247-263

15. https://www.ada.org/en/publications/adanews/2020-archive/march/ada-develops-guidanceon-dental-emergency-nonemergency-care).

16. Jampani ND, Nutalapati R, Dontula BSK, Boyapati R. Applications of teledentistry: A literature review and update, 4PMID: 24478952 PMCID: PMC3894070 DOI: 10.4103/22310762.97695

17. Centers for Disease Control and Prevention Discontinuation of Isolation for Persons with COVID-19 Not in Healthcare Settings. Washington, $\quad$ D.C.: 2020. "https://www.cdc.gov/coronavirus/2019

18. https://www.ada.org/ /media/CPS/Files/COVID/ ADA_Int_Guidance_Mgmt_Emerg-

Urg_Dental_COVID19?utm_source=adaorg\&utm _medium $=$ VanityURL\&utm_content=interimguid ance-flowcharts\&utm_campaign $=$ covid-19

19. Occupational Safety and Health Administration Dentistry Workers and Employers. Washingto, DC: United States Department of Labor 2020. "https://www.osha.gov/SLTC/covid19/dentistry.html". Accessed 4/5/2020.)

20. Centers for Disease Control and Prevention Guidance for Dental Settings. Interim Infection Prevention and Control Guidance for Dental Settings During the COVID-19 Response. Washington, D.C. 2020. "https://www.cdc.gov/coronavirus/2019ncov/hcp/dental-settings.html".

Accessed $7 / 14 / 2020$.
21. Kumar GA, Mohan R, Hiremutt DRP, Vikhram KB. COVID-19 Pandemic and Safe Dental Practice: Need of the hour. J of Indian Academy of Oral Medicine \& Radiology. 2020; 32(2); 164171.

22. Guidelines for Dental Professionals in Covid- 19 pandemic situation. May 2020. Available from: https://www.mohfw.gov.in/pdf/DentalAdvisoryF)

23. Lucaciu O, Tarczali D, Petrescu N. Oral Healthcare during the COVID-19 Pandemic. J Dent Sci. 2020 May 1; 1-4.

24. Kampf G, Todt D, Pfaender S. Persistence of Coronaviruses on Inanimate Surfaces and Their Inactivation with Biocidal Agents. J Hosp Infect. 2020; 104: 246-251.

25. Kariwa H, Fujii N, Takashima I. Inactivation of SARS coronavirus by means of povidone- iodine, physical conditions, and chemical reagents. Jpn J Vet Res. 2004; 52:105- 12.

26. Samaranayake LP, Reid J, Evans D. The Efficacy of Rubber Dam Isolation in Reducing Atmospheric Bacterial Contamination. ASDC J Dent Child. 1989; 56: 442-444.

27. American Dental Association ADA Interim Guidance for Minimising Risk for COVID-19 Transmission. Chicago, III: 2020

28. Kalenderian E, Xiao Y, Heiko Spallek H, Franklin A, Olsen G, Walji MJ. COVID-19 and Dentistry: Challenges and Opportunities for Providing Safe Care. PSNET. Aug 7, 2020.

29. The Bio-Medical Waste Management (Amendment) Rules, 2018. 2020. Available from: http://www.

indiaenvironmentportal.org.in/content/453336/ the- bio- $^{-}$medical- waste- management- amendm ent- ${ }^{-}$rules- 2018. 